"This is the peer reviewed version of the following article: Theobald, D., and Farrington, D. P. (2016) Advancing knowledge about dating violence. Crim Behav Ment Health, 26: 225-228. doi:

10.1002/cbm.2010., which has been published in final form at http://dx.doi.org/10.1002/cbm.2010. This article may be used for non-commercial purposes in accordance with Wiley Terms and Conditions for Self-Archiving." 


\section{Editorial: Advancing Knowledge about Dating Violence}

\section{Delphine Theobald and David P. Farrington}

Dating violence perpetration and victimisation has increased considerably in recent years and public health is increasingly concerned about the possible negative outcomes that have a significant impact on the individual and society. Adolescence and emerging adulthood are important developmental periods to address this issue particularly for those who may have experienced violence in their family of origin and are prone to risk taking behaviours such as drinking, drug use and other antisocial behaviours.

Adolescence and emerging adulthood (see Arnett, 2015) are particularly important times as they represent a period of change in the nature and importance of relationships with peers and potential dating partners. This is a time when individuals may test out what behaviours will and will not be tolerated and this experience may well impact on judgements and behaviours in later adult relationships particularly during cohabitation and marriage. Dating violence has clear implications for future intimate partner violence and the possible transmission of violence from one generation to the next.

It is therefore imperative that early intervention to prevent dating violence perpetration and victimisation is offered, based on knowledge about the risk factors for this behaviour. In order to advance knowledge about risk factors, prospective, longitudinal studies are needed which investigate the development of dating violence in a number of different samples. This special issue brings together a wealth of findings from some major studies in the UK and the US; the Cambridge Study in Delinquent Development (CSDD; Farrington et al. 2009), the Pittsburgh Girls Study (PGS; Keenan et al., 2010), the Toledo Adolescent Relationships Study (TARS; Giordano et al., 2010), the Pathways to Desistance Study (PDS; Mulvey et al., 2004), the Rochester Youth Development Study (RYDS; Thornberry et al., 2003) and the Lehigh Longitudinal Study (LLS; Herrenkohl et al., 1991).

Previous research on possible risk factors for violence in intimate relationships using the CSDD (Theobald and Farrington, 2012) suggests that various individual factors measured in childhood and adolescence predict the perpetration of intimate partner violence in the second generation males (G2). The first study in this special issue focuses on dating violence in the third generation of the CSDD (G3), namely the sons and daughters of the original men. Delphine Theobald and colleagues examine the prevalence of dating violence compared with violence in cohabiting relationships in this community sample of males and females. They 
also address to what extent child and adolescent explanatory factors from the individual, family, parental and socio-economic domains predict this behaviour. Using the strongest risk factors they then determine a risk score for dating violence that might form a basis for future research on the assessment and prediction of this form of violence. The findings support the prior literature with regard to the extent to which females are more often the perpetrators of violence in both dating and cohabiting relationships, but the most important risk factors differ by gender.

The second paper in this issue, by Lia Ahonen and Rolf Loeber, focuses on one of the possible mechanisms for dating violence perpetration and victimisation in girls. The literature on dating violence perpetration and victimisation suggests that girls seem to experience more long term negative outcomes than boys. In this very interesting paper, Ahonen and Loeber examine parental emotional regulation as a possible mechanism that might influence both perpetration and victimisation in girls, utilising data from the PGS. The authors found, when controlling for race and early aggression, daughters who had a parent who had high levels of negative emotion were more likely to be involved in serious dating violence than those experiencing more positive emotion regulation in their parents. This is an important finding and sheds further light on the complex mechanisms involved.

A major concern with any act of violence, and particularly dating violence, is the implication for later health outcomes. There are numerous mental and physical health consequences of intimate partner violence reported in the literature but the majority of this research is based on clinical samples of women in cohabiting relationships including marriage. Thus it is not clear if young females and males experience similar physical health outcomes when they are exposed to dating violence. Jennifer Copp and colleagues address this issue in the next paper in this special issue. They examined the direct association between dating violence and self-rated physical health during adolescence and across the transition to adulthood using information from the TARS, taking into account potential mediating and confounding factors such as exposure to childhood abuse, correlates of physical and emotional well-being, and lifestyle characteristics. Consistent with prior research on the association between intimate partner violence and physical health, they show that dating violence is associated with a decline in self-rated physical health from adolescence through to early adulthood among both males and females.

Whether risk factors for dating violence are similar or different across particular populations is also worthy of investigation. Clearly, it is important to consider to what extent risk factors identified in community samples as predictors of dating violence also apply to 
vulnerable populations such as high risk youth. Gary Sweeten and his colleagues address this issue using information from the PDS, a sample of high risk youth, modelling group-based trajectories based on emotional and physical dating violence perpetration. The authors were able to identify three developmental patterns for emotional dating violence and physical dating violence: a non-offending group, a low-level offending group and a chronic high-level offending group. However, the prevalence in each group differed by the type of violence perpetrated. They found that psychosocial traits distinguished between emotional and physical violence across the trajectory groups with the high-violence groups having serious psychosocial deficits across a range of measures. The authors conclude that their findings might suggest earlier points for intervention to reduce later violence.

In view of the identification of numerous risk factors across a variety of domains that might be implicated in dating violence it is important to consider the potential for risk assessment. Risk assessment for dating violence is an under-developed area of research. In the next paper in this special issue James Tapp and Estelle Moore offer a ground-clearing review of the literature based on individuals aged 15-30 years. These authors assessed the predictive validity, accuracy and inter-rater reliability of risk assessments utilised so far. This thorough systematic review found only five studies that described assessments that focussed specifically on risk factors for dating violence. The studies indicate that young people are at greater risk of being involved in dating violence if they have experienced violence in other attachment relationships and have limited coping skills. The authors also found that having peers with positive attitudes to violence was an important factor. Clearly, further investigation is warranted in order to develop specific dating violence instruments and this paper creates a foundation for future research in this area.

The next paper in this issue investigates the continuity of relationship violence over a period of 8-10 years. Specifically, Sarah Greenham and Mauri Matsuda assess to what extent dating violence in early adulthood is associated with later intimate partner violence, using data from the RYDS. The findings suggest that there is a relationship between dating violence and later violence in adult intimate relationships. While four protective factors (family support, family attachment, peer support and parental support) predicted low rates of adult intimate partner violence, none of these reduced the continuity over time.

Our last paper utilises data from the Lehigh Longitudinal Study. Todd Herrenkohl and Hyunzee Jung investigate whether different forms of violence experienced in particular 
developmental periods are influential predictors in relationship violence in adulthood. The authors found that child maltreatment and adolescent dating violence victimisation predicted later adult intimate partner violence victimisation and perpetration.

Dating violence is clearly a very important topic but the mechanisms leading to both perpetration and victimisation are complex. The findings from these important longitudinal studies highlight the necessity for early intervention based on risk factors from a number of domains. It is also clear that there are similarities between the risk factors and outcomes from the general violence risk literature and those for dating violence. Risk assessment should therefore not necessarily focus on one type of behaviour but on general violence risk. We hope that these articles help to advance knowledge about dating violence and ultimately help to develop effective prevention techniques.

\section{References:}

Arnett JJ (2015) Emerging adulthood: The winding road from the late teens through the twenties. New York: Oxford University Press.

Giordano PC, Soto DA, Manning WD, Longmore MA (2010) The characteristics of romantic relationships associated with teen dating violence. Social Science Research 39: 863-874.

Farrington DP, Coid JW, West DJ (2009) The development of offending from age 8 to age

50: Recent results from the Cambridge Study in Delinquent Development. Monatsschrift fur Kriminologie und Strafrechtsreform (Journal of Criminology and Penal Reform) 92: 160-173.

Herrenkohl RC, Herrenkohl EC, Egolf BP, Wu P (1991) The developmental consequences of child abuse: The Lehigh Longitudinal Study. In: Starr RH , Wolfe DA (eds) The effects of child abuse and neglect: Issues and research. New York: The Guilford Press pp. 57-81.

Keenan K, Hipwell A, Chung T, Stepp S, Stouthamer-Loeber M, Loeber R, McTigue K (2010) The Pittsburgh Girls Study: Overview and initial findings. Journal of Clinical Child and Adolescent Psychology 39: 506-521.

Mulvey EP, Steinberg L, Fagan J, Cauffman E, Piquero AR, Chassin L, Knight GP, Brame, R, Schubert CA, Hecker T, Losoya SH (2004) Theory and research on desistance 
from antisocial activity among serious adolescent offenders. Youth Violence and Juvenile Justice 2:213-236.

Theobald D, Farrington DP (2012) Child and adolescent predictors of male intimate partner violence. Journal of Child Psychology and Psychiatry 53:1242-1249.

Thornberry TP, Lizotte AJ, Krohn MD, Smith CA (2003) Causes and consequences of delinquency: Findings from the Rochester Youth Development Study, In Thornberry TP, Krohn MD (eds) Taking Stock of Delinquency. New York:Kluwer Academic/Plenum pp.11-46. 